\title{
Hirnveränderungen nicht medikationsbedingt
}

Fragestellung: Lassen sich bei Patienten, die langjährig unter einer schizophrenen Erkrankung leiden, bisher aber noch nie medikamentös behandelt worden sind, spezifische morphologische Auffälligkeiten des Gehirns feststellen?

Hintergrund: In vielen früheren Untersuchungen musste letztlich offen bleiben, ob die beschriebenen Auffälligkeiten Folge der Erkrankung oder einer langjährigen Medikation waren.

Patienten und Methodik: Mithilfe von T1-gewichteten, hochauflösenden MRT-Schnitten wurden 25 Patienten, die seit fünf bis 47 Jahren unter einer schizophrenen Erkrankung litten und bisher nicht medikamentös behandelt worden waren, im Vergleich zu 33 gesunden Kontrollpersonen untersucht. Der Durchmesser des Kortex und das Volumen der grauen Substanz wurden verglichen. Im Fall von signifikanten Gruppenunterschieden wurde unter Berücksichti-
Zhang W, Deng W, Yao L et al. Brain structural abnormalities in a group of never-medicated patients with long-term schizophrenia. Am J Psychiatry 2015; 172: $995-1003$ gung von altersabhängigen Effekten untersucht, ob sich in der Gruppe der Patienten mit schizophrenen Erkrankungen eine signifikant stärkere Abnahme der genannten Parameter nachweisen lässt.
Ergebnisse: In der Gruppe der schizophrenen Patienten fiel in mehreren Hirnarealen eine signifikant verminderte Kortexdicke auf (ventromedialer präfrontaler Kortex, linker Gyrus temporalis superior, Pars triangularis rechts). Die Auswertung ergab Hinweise auf eine beschleunigte altersabhängige Verdünnung der kortikalen Schichten in den genannten Hirnregionen, während dieser Prozess im Bereich des linken Globus parietales superior eher verlangsamt erschien.

Auch im Hinblick auf das Volumen der grauen Substanz ergab sich ein differenziertes Bild: höhere Werte im Bereich des Putamens beidseits und geringere Volumina im rechten Gyrus temporalis, wenn die Gruppe der Patienten mit der Kontrollgruppe verglichen wurde. Eine Veränderung der altersbedingten Verschiebungen konnte im Hinblick auf die graue Substanz nicht festgestellt werden.

Schlussfolgerungen: Das Hauptergebnis dieser Untersuchung, nämlich die beschleunigte altersabhängige Abnahme der präfrontalen und temporalen kortikalen Schichten deuten darauf hin, dass es im Rahmen einer schizophrenen Erkrankung zu progressiven neurodegenerativen Prozessen kommt, die aber auf spezifische Hirnregionen beschränkt bleiben und die nicht auf eine langjährige medikamentöse Behandlung zurückgeführt werden können.

\section{- Kommentar von Andreas Broocks, Schwerin}

\section{Schizophrenien gehen mit neurodegenerativen Veränderungen einher}

Die Hypothese, dass schizophrene Erkrankungen mit fortschreitenden neurodegenerativen Prozessen des Gehirns verbunden sind, wurde bereits von Emil Kraepelin vertreten und ist bis heute wissenschaftlich hochaktuell. Immer wieder wurde die Frage aufgeworfen, ob hirnmorphologische Auffälligkeiten nicht durch die langjährige Zufuhr antipsychotisch wirksamer Substanzen bedingt ist.

Die große Bedeutung der vorliegenden Arbeit ergibt sich daraus, dass es der chinesischen Arbeitsgruppe gelungen ist, ein vergleichsweise großes Kollektiv von Patienten zu finden und zu untersuchen, die trotz langer Krankheitsdauer noch nicht medikamentös behandelt worden waren. Die Auswertung ergab ein durchaus differenziertes Bild: Während der aus früheren Untersuchungen bekannte Befund einer signifikant verminderten Kortexdicke bestätigt wurde, fanden sich bezüglich der grauen Substanz im Bereich des Putamens beidseits erhöhte Werte in der Patientengruppe.

Die Autoren diskutieren als mögliche Ursache kompensatorische Prozesse, die mit einer erhöhten neuronalen und metabolischen Aktivität im Bereich des Striatums einhergehen. Aufgrund der hohen Variabilität im Hinblick auf das Alter und die Krankheitsdauer der Patienten ist es möglich gewesen, eine Akzeleration der altersbedingten Abnahme der präfrontalen und temporalen Kortexdicke nachzuweisen.
Die Autoren räumen selbst ein, dass eine prospektiv angelegte longitudinale Studie im Vergleich zur vorliegenden Querschnittsuntersuchung wesentlich aussagekräftiger wäre, weisen aber zu Recht auf die praktische Undurchführbarkeit einer solchen wissenschaftlich idealen Studie hin.

Zusammenfassend kann gesagt werden, dass diese qualitativ hochwertige Untersuchung aufgrund der großen Gruppe von an Schizophrenie erkrankten aber medikamentös unbehandelten Menschen einen wichtigen Beitrag zum Verständnis der Pathogenese dieser Erkrankung darstellt.

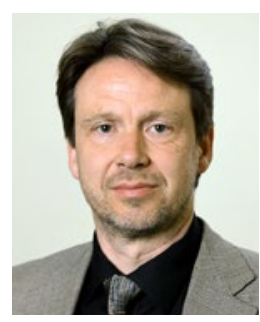

Prof. Dr. med. Andreas Broocks, Schwerin

Carl-Friedrich-Flemming-Klinik

HELIOS Kliniken Schwerin

E-Mail: andreas.broocks@helios-kliniken.de 